\title{
Saliva as Alternative to Naso-Oropharyngeal Swab for SARS- CoV-2 Detection by RT-qPCR: A Multicenter Cross-Sectional Diagnostic Validation Study
}

\section{Michael L. Tee ( $\sim$ mltee@up.edu.ph ) \\ University of the Philippines Manila, National Capital Region}

\section{Aedrian A. Abrilla}

University of the Philippines Manila, National Capital Region

\section{Cherica A. Tee}

University of the Philippines Manila, National Capital Region

\section{Leslie Michelle M. Dalmacio}

University of the Philippines Manila, National Capital Region

Vivencio Jose P. Villaflor

Dagupan Doctors Villaflor Memorial Hospital

\section{Al-Zamzam A. Abubakar}

Ciudad Medical Zamboanga

\section{Pedrito Y. Tagayuna}

Fe Del Mundo Medical Center

\section{Sheldon Steven C. Aquino}

Dagupan Doctors Villaflor Memorial Hospital

Vicente Aaron L. Bernardo VI

Dagupan Doctors Villaflor Memorial Hospital

\section{Ronald R. Matias}

St. Luke's Medical Center

\section{Research Article}

Keywords: Coronavirus disease 2019 (COVID-19), naso-oropharyngeal swab, Philippines, saliva, SARS-CoV-2

Posted Date: February 22nd, 2022

DOl: https://doi.org/10.21203/rs.3.rs-1350489/v1

License: (c) (i) This work is licensed under a Creative Commons Attribution 4.0 International License. Read Full License

Version of Record: A version of this preprint was published at Scientific Reports on July 23rd, 2022. See the published version at https://doi.org/10.1038/s41598-022-16849-1. 


\section{Abstract}

Saliva has been demonstrated as feasible alternative to naso-oropharyngeal swab (NOS) for SARS-CoV-2 detection through reverse transcription quantitative/real-time polymerase chain reaction (RT-qPCR). This study compared the diagnostic agreement of conventional NOS, saliva with RNA extraction (SE) and saliva direct without RNA extraction (SalivaDirect) processing for RT-qPCR in identifying SARS-CoV-2. All techniques were also compared as separate index tests to a composite reference standard (CRS) where positive and negative defined as SARS-CoV-2 detection in either one and no sample, respectively. Of 518 paired samples, SARS-CoV-2 was detected in 150 (28.96\%) NOS and 151 (29.15\%) saliva specimens. The saliva-based tests were noted to have a sensitivity, specificity and accuracy (95\% confidence interval) of $92.67 \%$ ( $87.26 \%$, 96.28\%), 97.55\% (95.41\%, 98.88\%) and 96.14\% (94.10\%, 97.63\%), respectively, for SE RT-qPCR and 90.67\% (84.84\%, 94.80\%), 98.91\% (97.24\%, 99.70\%) and 96.53\% (94.56\%, 97.93\%), respectively, for SalivaDirect RT-qPCR compared to NOS RT-qPCR. Compared to CRS, all tests demonstrated diagnostic performance that is statistically similar to each other. These suggest that both conventional and streamlined saliva RT-qPCR are non-inferior to conventional NOS RT-qPCR in detecting SARS-CoV2.

\section{Introduction}

Integral to the control of the coronavirus disease 2019 (COVID-19) pandemic is accurate, adequate, and timely detection of severe acute respiratory syndrome coronavirus 2 (SARS-CoV-2) infections [1]. Identification of specific combinations of SARSCoV-2 ribonucleic acid (RNA) genomic elements through reverse transcription quantitative/real-time polymerase chain reaction (RT-qPCR) has been the internationally accepted reference testing platform to this end, with naso-oropharyngeal swab (NOS) being the most commonly preferred patient sample [2]. As the algorithm from sample collection to testing in this prevailing paradigm is rife with economic, logistical and personnel safety issues [3, 4], we need to establish evidence-based, streamlined workflow that maintains reasonable diagnostic accuracy. This gains more importance in resource-disadvantaged developing nations that may disproportionately bear the brunt of the pandemic, such as the Philippines. With a peak test positivity rate of almost $50 \%$, the country has more than 3.5 million total cases, roughly 230,000 active cases and over 53,000 deaths [5]. It is second only to Indonesia in terms of absolute COVID-19 burden among Southeast Asian nations [6].

The evidence of saliva having diagnostic utility that is equivalent to NOS for detecting SARS-CoV-2 has since grown substantially to professional and public acceptance [7, 8]. Considering the potential to additionally reduce local testing costs and requirements, we endeavored to comprehensively evaluate SalivaDirect [9], an RNA extraction-free saliva sample processing technique, for detecting SARS-CoV-2 through RT-qPCR in the Philippine setting.

\section{Materials And Methods}

\section{Study Design and Ethics Review}

The protocol of this prospective cross-sectional study was reviewed and approved, with its implementation monitored, by the ManilaMed Ethics Review Committee (MMERC No. 2021-06). The study was performed in accordance with the Declaration of Helsinki and the International Ethical Guidelines for Biomedical Research involving human subjects.

\section{Participants}

Volunteers considered potentially eligible for inclusion and eventually invited to participate, through consecutive sampling, were inpatient or outpatient adults availing NOS RT-qPCR SARS-CoV-2 testing, from May to December 2021, in one of three Philippine hospitals: Fe Del Mundo Medical Center (Quezon City, Metropolitan Manila, National Capital Region), Dagupan Doctors Villaflor Memorial Hospital (Dagupan City, Pangasinan Province, Luzon) and Ciudad Medical Zamboanga (Zamboanga City, Zamboanga del Sur, Mindanao). The volunteers were included if they can give informed consent and to autonomously collect drooled saliva. Volunteers were excluded if they were unable to ensure avoidance of enteral intake, 
gargling with mouthwash or brushing teeth, and smoking for at least 30 minutes before providing saliva. Demographic and clinical data such as history of exposure and symptoms were obtained from consenting participants.

\section{Test Methods}

Paired samples of saliva and NOS were collected from all volunteers. The samples of each patient were placed in separate code-labeled tubes (one for NOS and one for saliva), and these tubes were stored for transport in containers distinctively carrying only either type of sample. These were subjected to subsequent laboratory procedures within 24 hours from sample collection, with separation in terms of personnel and instruments based on sample type. Interpretation of the tests was done independently by two assessors who did not have access to clinical information, and the result from the counterpart sample of the same study volunteer. The samples were processed and tested in laboratories using harmonized protocols in the aforementioned hospitals that are licensed to operate SARS-CoV-2 RT-qPCR facilities by the Philippine Department of Health.

\section{Saliva RT-qPCR}

Prior to NOS sampling, included volunteers were asked to drool at least $1 \mathrm{~mL}$ of saliva in a $5 \mathrm{~mL}$ sterile tube. The filled sterile tube was then sealed and stored in a chest at room temperature before transport to the designated laboratory. Each saliva sample was partitioned for conventional saliva RNA extraction (SE) and SalivaDirect procedures. The volume allotted for SE was subjected to manufacturer-prescribed conditions through the Nextractor NX-48 automated system (Genolution Inc., South Korea). Sample preparation for SalivaDirect was performed following the method described by Vogels et al. [9]. In brief, $2.5 \mu \mathrm{L}(50 \mathrm{mg} / \mathrm{mL})$ of Proteinase $\mathrm{K}$ was added to $50 \mu \mathrm{L}$ saliva in PCR tubes, which were then vortexed at 3200 revolutions per minute for 1 minute. The samples were then heated at $95^{\circ} \mathrm{C}$ for 5 minutes. From these RNA-extracted and SalivaDirectprocessed mixtures, $5 \mu \mathrm{L}$ was utilized as input in subsequent RT-qPCR processes to amplify SARS-CoV-2 RNA-dependent RNA polymerase $(R d R p)$, envelope $(E)$, and nucleocapsid $(M$ genes using the GeneFinder COVID-19 Plus RealAmp Kit (OSANG Healthcare Co., Ltd., South Korea). The kit utilizes human RNase $P$ gene template as internal control, and $R d R p, E, N$ and $R$ Nase $P$ amplified constructs in RT-qPCR are dyed with fluorescein amidite (FAM), Texas Red, 5'-dichloro-dimethoxyfluorescein/Victoria (JOE/VIC), and Cy5 fluorophores, respectively. An RT-qPCR run was deemed valid if (1) the quantitation cycle (Cq) readings for $R d R p, E, N$ and $R N$ ase $P$ were all $\leq 22.00$ for the designated positive controls and (2) the negative control $\mathrm{Cq}$ readings for the same genes were all $\geq 40.00$ or blank/undetermined. A sample was considered positive for SARSCoV-2 if the Cq readings for $R N A$ se $P$ and at least either $R d R p, E$ or $N$ were $\leq 40.00$, with the characteristic sigmoidal amplification curve noted in all instances. A negative result for SARS-CoV-2 was indicated if $R$ Nase $P$ Cq $\leq 40.00$ with sigmoidal amplification curve and Cq readings for $R d R p, E$ and $N$ were all $\geq 40.00$ or blank/undetermined. Sample retesting was immediately performed if $R N$ ase $P C q \geq 40.00$ regardless of $R d R p, E$ and $N C q$ values. All test reactions were maintained in the CFX96 RT-qPCR system (Bio-Rad Laboratories, California, United States of America).

\section{NOS RT-qPCR}

The conventional RNA extraction-dependent RT-qPCR technique to detect SARS-CoV-2 in NOSs was utilized to evaluate the utility of the saliva-based tests. Volunteers underwent naso-oropharyngeal swabbing performed by trained personnel, and the swabs were then placed in sterile tubes containing universal transport medium. The filled tubes were sealed and stored at a $4-6^{\circ} \mathrm{C}$ cold chest before transport to the designated laboratory. Manufacturer-prescribed instructions were followed for sample preparation through the Nextractor NX-48 automated system and RT-qPCR steps. The criteria for RT-qPCR run validity and NOS sample positivity were the same as those for saliva samples.

\section{Data Analysis}

The Buderer technique [10] was used to compute minimum sample size. Presuming the prevalence of COVID-19 at 10\% during the period of study planning, a target sensitivity and specificity of at least $90 \%$ for saliva-based RT-qPCR compared to NOS RT-qPCR, and the significance level (a) and maximum acceptable width of the $95 \%$ confidence interval (Cl) being set at 0.05 and $10 \%$, respectively, yielded a minimum sample size of 385 . 
Descriptive statistics were presented as proportions for categorical variables and mean (standard deviation or SD) or median (interquartile range or IQR) for continuous variables. Diagnostic validity estimates (sensitivity, specificity, positive predictive value or PPV, negative predictive value or NPV, and accuracy) and their $95 \%$ Cls were calculated, using the MedCalc online calculator [11], for three scenarios: (1) SalivaDirect RT-qPCR as index test and NOS RT-qPCR as reference test, (2) SE RT-qPCR as index test and NOS RT-qPCR as reference test, and (3) SalivaDirect, SE and NOS RT-qPCR as index tests separately benchmarked against a composite reference standard (CRS). In the CRS, a participant with at least either a SARS-CoV-2detectable NOS or SalivaDirect or SE sample was considered positive and a volunteer with all samples without SARS-CoV-2 gene detection as negative for the virus [12]. McNemar Chi-square test was used to statistically compare the specified indexreference test pairings. Cohen Kappa $(\mathrm{K})$ coefficients for agreement between the designated index and reference tests were also estimated [13]. A post hoc analysis comparing the mean (standard deviation or SD) Cq values of SARS-CoV-2 genes between NOS, SalivaDirect and SE samples among volunteers positive in all was also conducted through repeated-measures analysis of variance with Tukey's honest significant difference test as post hoc test. Statistical significance was set at $p$ $\leq .0500$ unless otherwise specified.

\section{Results}

A total of 518 adults provided paired NOS and saliva samples for SARS-CoV-2 testing (Figure). The median (IQR) age of the entire sample was $31.5(26-43)$ years and $224(43.24 \%)$ were female. A total of $150(28.96 \%)$ NOS and $151(29.15 \%)$ saliva samples were positive for SARS-CoV-2, while 160 (30.89\%) participants had at least one positive sample. Among those with a positive saliva sample, SARS-CoV-2 was detected in 148 (28.57\%), 140 (27.03\%) and 137 (26.45\%) participants whose specimens were processed under SE, SalivaDirect and both, respectively. The resulting contingency table comparing SE RTqPCR with conventional NOS RT-qPCR as reference (Table 1) revealed a sensitivity of $92.67 \%$ (95\% Cl: $87.26 \%, 96.28 \%$ ), specificity of $97.55 \%$ (95\% Cl: $95.41 \%, 98.88 \%$ ) and accuracy of $96.14 \%(95 \% \mathrm{Cl}: 94.10 \%, 97.63 \%)$. On the other hand, comparing SalivaDirect RT-qPCR to the swab-based test yielded a sensitivity of $90.67 \%$ (95\% Cl: $84.84 \%, 94.80 \%)$, specificity of $98.91 \%$ (95\% Cl: $97.24 \%, 99.70 \%)$ and accuracy of $96.53 \%$ (95\% Cl: $94.56 \%, 97.93 \%)$. The Cohen $\mathrm{k}$ coefficient estimate for agreement between the SE and swab tests is $0.82(95 \% \mathrm{Cl}: 0.75,0.90)$ while that between the SalivaDirect and swab tests is 0.84 (95\% Cl: 0.77, 0.91), both suggesting almost perfect agreement. When NOS RT-qPCR, SE RT-qPCR and SalivaDirect RTqPCR were individually compared to the CRS, the swab test showed the numerically highest estimates for sensitivity, NPV, accuracy and Cohen $\mathrm{k}$ followed by SE RT-qPCR and then by SalivaDirect RT-qPCR (Table 2). Nevertheless, these differences are unlikely to be statistically significant. Comparing the paired samples (NOS, SE and SalivaDirect) that were all positive for SARS-CoV-2 showed statistically significant difference in mean (SD) Cq values for all SARS-CoV-2 gene targets $(p<.0001$ in all comparisons). NOS samples have significantly lower mean Cq than both SE and SalivaDirect for SARS-CoV-2 RdRp, Nand E genes, while both saliva samples have significantly lower mean Cq than NOS for human RNase P gene (Table 3) 
Table 1

Diagnostic agreement contingency table and estimates comparing SE and SalivaDirect RT-qPCR, as separate index tests, to NOS RT-qPCR for SARS-CoV-2 detection.

\begin{tabular}{|c|c|c|c|c|c|c|c|c|c|}
\hline \multicolumn{10}{|c|}{ NOS RT-qPCR } \\
\hline SE & & Positive & Negative & Total & Saliva & & Positive & Negative & Total \\
\hline \multirow[t]{9}{*}{ RT-qPCR } & Positive & 139 & 9 & 148 & Direct & Positive & 136 & 4 & 140 \\
\hline & Negative & 11 & 359 & 370 & RT-qPCR & Negative & 14 & 364 & 378 \\
\hline & Total & 150 & 368 & 518 & & Total & 150 & 368 & 518 \\
\hline & & \multicolumn{3}{|c|}{ PE $(95 \% \mathrm{Cl})$} & & & \multicolumn{3}{|c|}{$\mathrm{PE}(95 \% \mathrm{Cl})$} \\
\hline & Sn & \multicolumn{3}{|c|}{$92.67 \%(87.26 \%, 96.28 \%)$} & & Sn & \multicolumn{3}{|c|}{$90.67 \%(84.84 \%, 94.80 \%)$} \\
\hline & $\mathrm{Sp}$ & \multicolumn{3}{|c|}{$97.55 \%(95.41 \%, 98.88 \%)$} & & $\mathrm{Sp}$ & \multicolumn{3}{|c|}{$98.91 \%(97.24 \%, 99.70 \%)$} \\
\hline & PPV & \multicolumn{3}{|c|}{$93.92 \%(89.00 \%, 96.72 \%)$} & & PPV & \multicolumn{3}{|c|}{$97.14 \%(92.76 \%, 98.90 \%)$} \\
\hline & NPV & \multicolumn{3}{|c|}{$97.03 \%(94.86 \%, 98.30 \%)$} & & NPV & \multicolumn{3}{|c|}{$96.30 \%(94.04 \%, 97.72 \%)$} \\
\hline & Accuracy & \multicolumn{3}{|c|}{$96.14 \%(94.10 \%, 97.63 \%)$} & & Accuracy & \multicolumn{3}{|c|}{$96.53 \%(94.56 \%, 97.93 \%)$} \\
\hline
\end{tabular}

Abbreviations: $\mathrm{Cl}$, confidence interval; NOS, naso-oropharyngeal swab; NPV, negative predictive value; PE, point estimate; RTqPCR, reverse transcription quantitative/real-time polymerase chain reaction; SE, saliva with RNA extraction; Sn, sensitivity; $\mathrm{Sp}$, specificity; PPV, positive predictive value.

Table 2

Diagnostic validity contingency table and parameter estimates comparing NOS RT-qPCR, SE RT-qPCR and SalivaDirect RTQPCR as separate index tests to the CRS (SARS-CoV-2 gene target detected in at least either one index test and not detected in all index tests considered as true positive and negative, respectively) for SARS-CoV-2 detection.

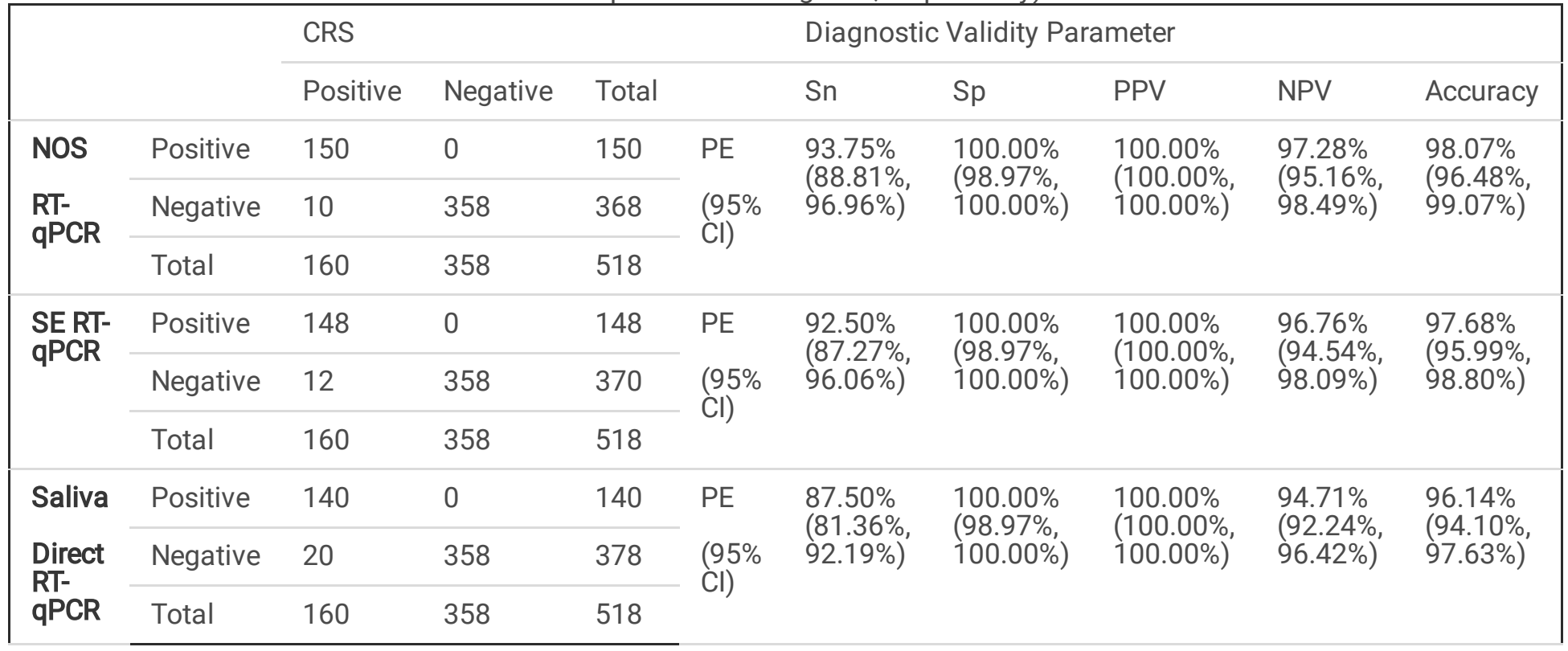

Abbreviations: $\mathrm{Cl}$, confidence interval; NOS, naso-oropharyngeal swab; NPV, negative predictive value; PE, point estimate; RTqPCR, reverse transcription quantitative/real-time polymerase chain reaction; SE, saliva with RNA extraction; Sn, sensitivity; Sp, specificity; PPV, positive predictive value. 
Table 3

RT-qPCR target gene $\mathrm{C}_{\mathrm{q}}$ value comparison of paired NOS, SE and SalivaDirect samples with positive readings $(\mathrm{n}=$ 137).

\begin{tabular}{|c|c|c|c|c|c|}
\hline \multirow[t]{2}{*}{ Gene (dye) } & \multicolumn{3}{|l|}{ Mean (SD) Cq } & \multirow[t]{2}{*}{ RM-ANOVA p-value } & \multirow{2}{*}{$\begin{array}{l}\text { Mean (SD) Cq } \\
\text { Statistical Ranking* }\end{array}$} \\
\hline & NOS & SE & SalivaDirect & & \\
\hline$R d R p(\mathrm{FAM})$ & $25.44(4.74)$ & $30.26(4.95)$ & $31.51(5.21)$ & $<.0001$ & NOS $<(\mathrm{SE} \approx$ SalivaDirect $)$ \\
\hline$N(\mathrm{JOE} / \mathrm{VIC})$ & $25.55(5.92)$ & $29.79(5.08)$ & $29.71(4.82)$ & $<.0001$ & NOS $<(\mathrm{SE} \approx$ SalivaDirect $)$ \\
\hline $\begin{array}{l}E \\
\text { (Texas Red) }\end{array}$ & $24.58(5.10)$ & $29.39(4.78)$ & $29.23(4.22)$ & $<.0001$ & NOS $<(\mathrm{SE} \approx$ SalivaDirect $)$ \\
\hline RNase $P$ (Су 5$)$ & $28.51(1.96)$ & $24.89(2.11)$ & $24.39(2.37)$ & $<.0001$ & NOS $>(S E \approx$ SalivaDirect $)$ \\
\hline
\end{tabular}

Abbreviations: Cq, quantitation cycle; $E$, envelope, FAM, fluorescein amidite; N, nucleocapsid; NOS, naso-oropharyngeal swab; $R d R p$, RNA-dependent RNA polymerase; RM-ANOVA, repeated-measures analysis of variance; SD, standard deviation; $\mathrm{SE}$, saliva with RNA extraction.

\section{Discussion}

Substantial evidence already exists on the non-inferiority of saliva relative to NOS as a specimen for conventional RNA extraction-dependent RT-qPCR detection of SARS-CoV-2 [7, 8]. Compared to NOS as a testing sample, the use of saliva has several advantages. In this alternative setup, compared to the number of staff that must be trained for adequate-quality NOS collection one patient at a time, fewer personnel needing less technical training are needed to facilitate simultaneous selfcollection of saliva by multiple patients. This potentially translates to a less aggregate risk of SARS-CoV-2 transmission between patients and personnel (due to avoidance of close-contact interactions secondary to swabbing, and fewer personnel contact with materials that could not be sanitized beforehand such as patient samples) and reduced demand for personal protective equipment (PPE). In terms of logistics, storage of samples before transport to the laboratory is less complicated (with the use of plain sterile vials rather than a combination of tubes, ice-cooled chest boxes, and proprietary swabs and transport reagents). Patients are also expected to be more amenable to such sample collection for SARS-CoV-2 testing than invasive and uncomfortable swabbing. It is also important to note that variation of several patient and personnel factors related to naso-oropharyngeal swabbing introduces iatrogenic preanalytical variability that may adversely affect testing results $[14,15]$. Furthermore, these findings may be useful in light of recent reports suggesting changing patterns of viral shedding, tissue tropism, and transmission mechanics [16-18], in favor of oral cavity and saliva, depending on the emerging SARS-CoV-2 variant such as Omicron [19].

Nevertheless, there remains fewer endeavors on attempting to streamline these laboratory processes without significant decline in analytical and/or diagnostic performance $[9,20]$. This is particularly important in the geographic and sociopolitical contexts of developing countries. Such settings are associated with challenges in addressing demands for COVID-19 pandemic response, with meager resources, such as sustaining testing coverage that is adequate, timely and accurate [21]. In the Philippines, a previous undertaking [22] adapted the RNA extraction-free technique, known as covidSHIELD, of the University of Illinois at Urbana-Champaign [20] for saliva RT-qPCR detection of SARS-CoV-2. This has led to the first regulatory approval of the technology in the country through Sansure Biotech (Hunan, China)-based molecular biology facilities of the Philippine Red Cross [23]. The present study, on the other hand, sought to evaluate the SalivaDirect procedure[9] through the harmonized laboratory system of a private hospital network, which utilizes GeneFinder reagents and Bio-Rad RT-qPCR platforms. While it may appear that covidSHIELD involves theoretically cheaper reagents for saliva processing (Tween 20 and Tris-Borate-Ethylenediaminetetraacetic acid [TBE] buffer) compared to SalivaDirect's Proteinase K, an advantage of the latter is that its heat-inactivation step is only 5 minutes or one-sixth of the time required by the other. On

Page 6/10 
top of the aforementioned benefits of the testing regime shift, from swab-based to saliva-based, streamlined pre-RT-qPCR saliva processing through heat inactivation and Proteinase K addition further reduces (1) the resource cost to release SARSCoV-2 RNA copies from capsids compared to the use of proprietary reagents, and (2) the risk of laboratory personnel exposure to viable SARS-CoV-2 units in patient samples (as the virus is known to be denatured at a fraction of time and thermal energy applied during the prescribed inactivation step [24]).

The results of the present study add to the growing body of evidence that indicate favorable comparability of saliva and NOS as specimens for SARS-CoV-2 nucleic acid-based detection. The head-on comparisons between SE RT-qPCR and NOS RTqPCR and between SalivaDirect RT-qPCR and NOS RT-qPCR reveal statistically similar diagnostic accuracy parameter estimates. Furthermore, our post-hoc analyses showed that among volunteers whose NOS, SE and SalivaDirect samples were also positive for SARS-CoV-2, the mean (SD) Cq values for all three SARS-CoV-2 gene targets ( $R d R p, N$ and $E)$ and the human RNase $P$ internal control are not significantly different between the SE and SalivaDirect specimens. As the Cq for a genomic target is inversely proportional to the initial amount of target gene copies in the post-processed RT-qPCR mixture, these findings imply that the presumed increase in heterogeneity of heat-inactivated and Proteinase K-treated saliva, compared to saliva that was processed conventionally, may not have adversely affected the analytical, diagnostic and quantitative performance of the RT-qPCR procedure common and downstream to both tests. However, compared to counterpart values for NOS, the SARS-CoV-2 viral load and the human cell content appear to be lower and higher, respectively, in both saliva specimen types. Higher $R d R p, N$ and $E$ Cq readings for NOS suggest that either (1) the viral load in the nasopharyngeal area is higher than in the oral cavity or (2) the liquid volume of saliva led to a "dilution" of the corresponding SARS-CoV-2 content. The significant discrepancy in human $R$ Nase $P$ Cq values may be due to the higher human cell content in saliva than in the nasopharyngeal swab scrapings.

Because volunteers having a positive saliva test result and a negative swab test result will be considered false positives in head-on comparison (with NOS RT-qPCR as the reference standard), the estimated specificity and PPV would have appeared to be lower than what could be expected in an RT-qPCR platform. Assuming that optimal quality control procedures were observed from test sample collection to processing, RT-qPCR specificity and PPV are theoretically 100\% (making it impossible to assign a sample as a false positive) because the primers designed to detect the genetic material of a pathogen is highly and molecularly specific to its genomic sequence [2]. It is thus more likely that saliva-positive but swab-negative volunteers represent truly-infected cases missed by swab testing, rather than false positives, and vice versa. Arguably, a false sense of security may befall such patients if they were tested only by NOS RT-qPCR. We hypothesize that this subset of the population may be a greater clinical or public health risk than their swab-positive but saliva-negative counterparts, considering that SARS-CoV-2, in terms of anatomy and biomechanics, is more likely to be released from the host to the environment (through aerosolization and droplet formation) in saliva than in the nasopharynx [25]. With this consideration of perfect specificity and PPV in mind, we then analytically constructed a CRS wherein the criterion for a positive case is at least either a SARS-CoV-2positive NOS, SD or SalivaDirect sample (virtually ensuring specificity and PPV of $100 \%$ since no volunteer can be considered false positive), and that for a negative case is SARS-CoV-2-negative NOS, SD and SalivaDirect samples. With this benchmark, the diagnostic performance of NOS RT-qPCR, SE RT-qPCR and SalivaDirect RT-qPCR was separately assessed. All tests performed statistically similar to one another in terms of sensitivity, NPV, accuracy and agreement in this scenario, again suggesting that both saliva as a sample and the streamlined technique to handle this specimen are non-inferior, at the very least, to the conventional test in epidemiologically detecting SARS-CoV-2. Another remarkable finding that is apparent in this construction is that all tests, even NOS RT-qPCR which is the considered reference standard, was not able to detect SARSCoV-2 in some volunteers whose other samples turned positive. While this can be due to iatrogenic factors (e.g., patientspecific and personnel-specific aspects concerning naso-oropharyngeal swabbing quality), potential pathophysiological and fluid-tissue localization mechanisms that are yet to be elucidated in SARS-CoV-2 infections may also be at play.

We recognize several limitations of this study. As a cross-sectional design was implemented, follow-up through clinical assessment and laboratory testing for at least one time point after initial study contact was not performed. Such a procedure could demonstrate viral kinetics in the specimens through the preclinical, clinical, and convalescent or mortality phases of the infection. This study was also insufficiently powered to determine the impact of sample collection timing (from the history of 
COVID-19 exposure and/or onset of relevant symptoms) on test results. RT-qPCR of NOS samples that underwent RNA extraction-free processing was also not performed; this would have provided direct information on the difference in categorical and quantitative $(\mathrm{Cq})$ results due to the change in pre-RT-qPCR processing technique for swab specimens. We also suggest that head-on comparison of the covidSHIELD and the SalivaDirect techniques be conducted to generate primary evidence on the relative performance and utility of these two major streamlining approaches for saliva samples. Nevertheless, considering the results of our study, we recommend the implementation of SalivaDirect for its potential to speed up testing turnaround time and to reduce laboratory costs.

\section{Declarations}

\section{ACKNOWLEDGMENTS}

The authors are deeply grateful to Mr. Rhais M. Gamboa, Executive Vice President and Chief Operating Officer of Mount Grace Hospitals, Inc, for his invaluable support to this study. The authors also thank Mr. Patrick Wincy C. Reyes of the School of Statistics, University of the Philippines Diliman for his statistical expertise.

\section{AUTHOR CONTRIBUTIONS}

MLT: Conceptualization, methodology, validation, formal analysis, investigation, resource, data curation, writing, supervision, project administration and funding acquisition; CAT: conceptualization, methodology, writing, supervision; LMMD:

conceptualization, methodology, writing, supervision; AAA: software, validation, data curation, formal analysis, writing; VJPV III: investigation, resource, site supervision, site project administration and funding acquisition; AZAA: investigation, resource, site supervision and site project administration, site data curation; PYT: investigation, resource, site supervision and site project administration, site data curation; SSCA: investigation, resource, site supervision and site project administration, site data curation; VALB VI: investigation, resources, supervision, site data curation; and

RRM: conceptualization, methodology, writing, supervision. All authors have read and agreed to the published version of this manuscript.

\section{DATA AVAILABILITY STATEMENT}

The corresponding author can provide the data upon request. It was submitted as part of the terminal report to the Medical Center Manila Ethics Review Board.

\section{COMPETING INTERESTS STATEMENT}

This endeavor was financially and logistically supported by Mount Grace Hospitals, Inc. which had no role in the design of the investigation and in the analysis of the results. Dr. Vivencio Jose P. Villaflor III is a member of the board of directors of Dagupan Doctors Villaflor Memorial Hospital. The proprietary instruments and materials used in this investigation are commonly and routinely utilized in our area of research and country, and the manufacturer of these products was not involved in any direct or indirect manner in the conduct of this research.

\section{References}

1. Cheng, M. P. et al. Diagnostic testing for severe acute respiratory syndrome-related coronavirus 2: a narrative review. Ann. Intern. Med. 172, 726-734 (2020).

2. Sethuraman, N., Jeremiah, S.S. \& Ryo, A. Interpreting diagnostic tests for SARS-CoV-2. JAMA. 323, 2249-2251 (2020).

3. Esbin M. N. et al. Overcoming the bottleneck to widespread testing: a rapid review of nucleic acid testing approaches for COVID-19 detection. RNA. 26, 771-783 (2020). 
4. Qian Y. et al. Safety management of nasopharyngeal specimen collection from suspected cases of coronavirus disease 2019. Int. J. Nurs. Sci. 7, 153-156 (2020).

5. COVID-19 Tracker. Department of Health (Philippines). https://www.doh.gov.ph/covid19tracker (2022).

6. Southeast Asian COVID-19 Tracker. Center for Strategic \& International Studies.

https://www.csis.org/programs/southeast-asia-program/southeast-asia-covid-19-tracker-0 (2022).

7. Bastos, M. L., Perlman-Arrow, S., Menzies, D. \& Campbell, J. R. The sensitivity and costs of testing for SARS-CoV-2 infection with saliva versus nasopharyngeal swabs: a systematic review and meta-analysis. Ann. Intern. Med. 174, 501510 (2021).

8. Butler-Laporte, G. et al. Comparison of saliva and nasopharyngeal swab nucleic acid amplification testing for detection of SARS-CoV-2: a systematic review and meta-analysis. JAMA Intern. Med. 181, 353-360 (2021).

9. Vogels, C. B. F. et al. SalivaDirect: a simplified and flexible platform to enhance SARS-CoV-2 testing capacity. Med. 2, 118 (2021)..

10. Buderer, N. M. F. Statistical methodology: I. Incorporating the prevalence of disease into the sample size calculation for sensitivity and specificity. Acad. Emerg. Med. 3, 895-900 (1996).

11. Diagnostic test evaluation calculator. MedCalc Software Ltd. [Internet]. https://www.medcalc.org/calc/diagnostic_test.php (2022).

12. Kandel, C. et al. Detection of SARS-CoV-2 from saliva as compared to nasopharyngeal swabs in outpatients. Viruses. 12, 1314 (2020).

13. Landis, J. R. \& Koch, G. G. The measurement of observer agreement for categorical data. Biometrics. 33, 159-174 (1977).

14. Lippi, G., Simundic, A.-M. \& Plebani, M. Potential preanalytical and analytical vulnerabilities in the laboratory diagnosis of coronavirus disease 2019 (COVID-19). Clin. Chem. Lab. Med. 58, 1070-1076 (2020).

15. Hiebert N. M., Chen, B. A. \& Sowerby, L. J. Variability in instructions for performance of nasopharyngeal swabs across Canada in the era of COVID-19 - what type of swab is actually being performed? J. Otolaryngol. Head. Neck. Surg. 50, 5 (2021).

16. Chu, C. Y. et al. Performance of saliva and mid-turbinate swabs for detection of the beta variant in South Africa. Lancet Infect. Dis. 21, 1354 (2021).

17. Huang N. et al. Integrated single-cell atlases reveal an oral SARS-CoV-2 infection and transmission axis. Preprint at https://www.medrxiv.org/content/10.1101/2020.10.26.20219089v1 (2020).

18. Lai, J. et al. Comparison of saliva and mid-turbinate swabs for detection of COVID-19. Preprint at: https://www.medrxiv.org/content/10.1101/2021.12.01.21267147v1 (2021).

19. Marais, G. et al. Saliva swabs are the preferred sample for Omicron detection. Preprint at: http://medrxiv.org/lookup/doi/10.1101/2021.12.22.21268246 (2021).

20. Ranoa, D. R. E. et al. Saliva-based molecular testing for SARS-CoV-2 that bypasses RNA extraction. Preprint at: http://biorxiv.org/lookup/doi/10.1101/2020.06.18.159434 (2020).

21. Chowdhury, A. Z. \& Jomo, K. S. Responding to the COVID-19 pandemic in developing countries: lessons from selected countries of the Global South. Development. 63, 162-171 (2020).

22. Tee, M. L. et al. Direct saliva versus conventional nasopharyngeal swab qRT-PCR to diagnose SARS-CoV2: validity study. Asian J. Res. Infect. Dis. 6, 37-46 (2021).

23. Right test for the right reason: updates on new testing technologies and protocols for COVID-19. Department of Health (Philippines). https://doh.gov.ph/doh-press-release/RIGHT-TEST-FOR-THE-RIGHT-REASON-UPDATES-ON-NEW-TESTINGTECHNOLOGIES-AND-PROTOCOLS-FOR-COVID-19 (2021).

24. Kampf, G., Voss, A. \& Scheithauer, S. Inactivation of coronaviruses by heat. J. Hosp. Infect. 105, 348-349 (2020)..

25. Xu, R. et al. Saliva: potential diagnostic value and transmission of 2019-nCoV. Int. J. Oral. Sci. 12, 11 (2020).

Page 9/10 


\section{Figures}

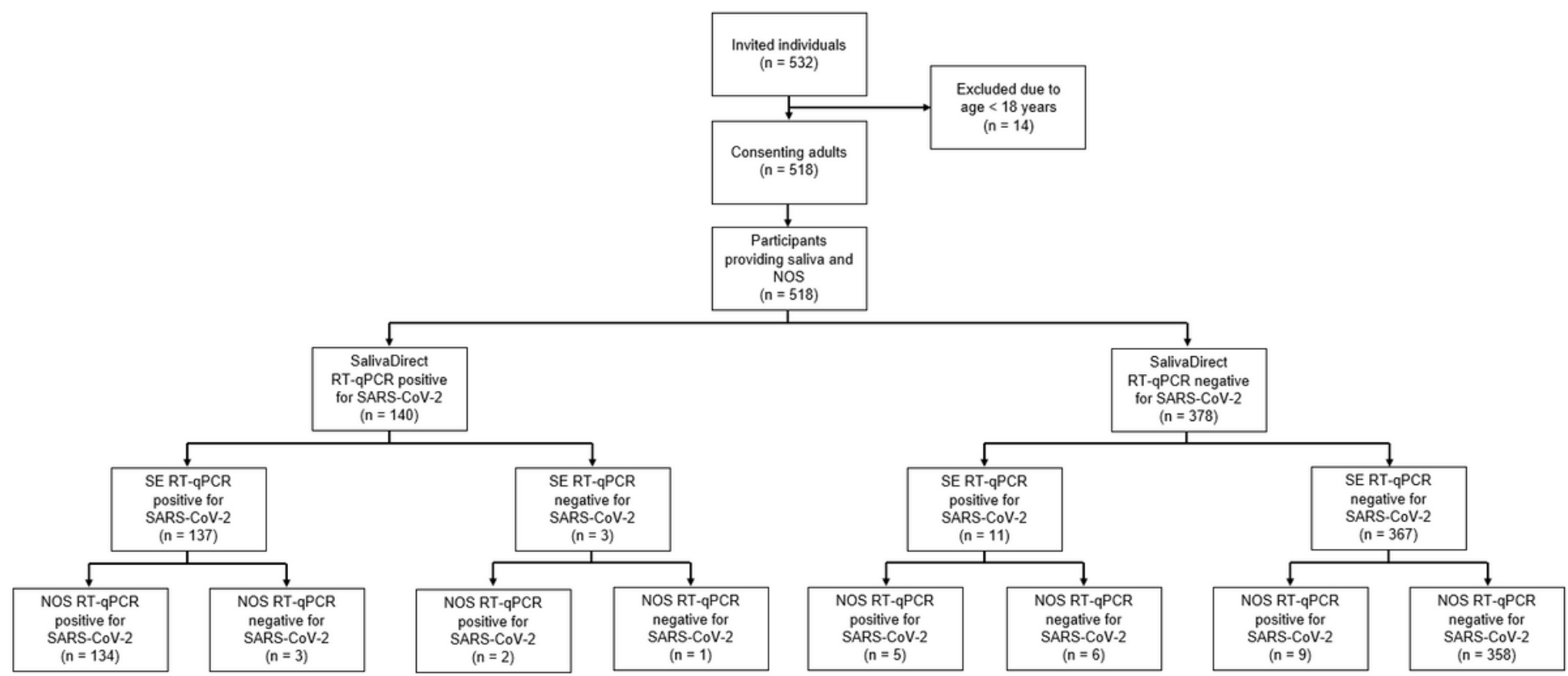

\section{Figure 1}

Flow diagram of participants in the study.

Abbreviations: NOS, naso-oropharyngeal swab; RT-qPCR, reverse transcription quantitative/real-time polymerase chain reaction; SARS-CoV-2, severe acute respiratory syndrome coronavirus 2; SE, saliva with RNA extraction. 